Gut, 1986, 27, 300-308

\title{
Simultaneous measurement of gastric emptying, small bowel residence and colonic filling of a solid meal by the use of the gamma camera
}

\author{
N W READ, M N AL-JANABI, A M HOLGATE, D C BARBER, AND C A EDWARDS \\ From the Departments of Physiology and Medical Physics, The University, Sheffield
}

SUMmaRY A method for determining the profiles of gastric emptying, small intestinal residence, and colonic filling of a solid test meal, labelled with $250 \mu \mathrm{Ci}{ }^{99}$ Technetium sulphur colloid has been evaluated in nine healthy volunteers and six patients with a disturbance in bowel habit. Mean small bowel transit time was determined by deconvolving the rate of colonic filling with the rate of gastric emptying. In normal subjects, the stomach appeared to empty exponentially with a half time of $1 \cdot 2 \pm 0 \cdot 3$ hours (mean $\pm S D$ ). Food reached the colon by $2 \cdot 8 \pm 1 \cdot 5$ hours. The mean small bowel transit time was $4.0 \pm 1.4$ hours. In most normal subjects, the colon appeared to fill in a linear fashion with approximately $16 \%$ food residues entering every hour, and the profile of colonic filling in normal subjects was similar to the profile of ileal emptying observed after feeding a similar radiolabelled solid meal to 14 patients equipped with terminal ileostomies. There was a highly significant correlation between the onset of breath hydrogen excretion and the appearance of radioactivity over the caecum $(r=0 \cdot 88, p<0 \cdot 01)$, though in one third of subjects the increase in caecal radioactivity preceded the rise in breath hydrogen concentration by more than 20 minutes. There was also a highly significant correlation between the mean transit time and values for colonic filling but not values for gastric emptying. Patients with irritable bowel syndrome who had diarrhoea tended towards short small bowel transit and early colonic filling, whereas patients who have constipation tended towards long small bowel transit and delayed colonic filling. This method offers a novel means of assessing small bowel transit time, small bowel residence and the profile of colonic filling in man.

The possible importance of the small bowel transit time in regulating absorption or digestion in man, and the factors that control small bowel transit are poorly understood. Part of the reason for this is that there is no good method that reliably and accurately measures the transit of food through the small intestine. The breath hydrogen technique ${ }^{12}$ can accurately determine the time that the head of the meal reaches the caecum but gives little information on the dynamics of entry of the remainder of the meal to the colon, and the average transit time through the small intestine. Measuring the transit of barium through the small intestine during the standard follow through examination of a barium meal $^{34}$ does not necessarily give any impression of the rate of passage of food through the small

Address for correspondence: Dr N W Read, Clinical Research Unit, H Floor, Royal Hallamshire Hospital, Sheffield S10 2JF.

Received for publication 24 June 1985. intestine. Mixing the food with barium and plotting its progress through the bowel on a television screen after image intensification provides a more accurate index of the rate of entry of food residues into the colon, ${ }^{5}$ but the radiation exposure is unacceptable and the large amount of barium needed may well interfere with the normal rate of passage of food through the intestine. We have used the gamma camera to monitor the passage of a labelled solid meal through the stomach and small intestine, and by computer analysis of the stored images, we have obtained profiles of gastric emptying and colonic filling, from which we have calculated values for the residence of food in the small intestine and the mean small bowel transit time. These data have been compared with breath hydrogen profiles obtained from the same subjects and with profiles of the delivery of the residues of a similar solid meal in patients with terminal ileostomies. 
Methods

\section{SUBJECTS}

Studies were carried out in nine normal male volunteers (aged between 20 and 26 years), who had no history of gastrointestinal illness and were taking no medication.

Studies were also carried out on six patients (two men and four women, aged between 28 and 43 years) who presented with bowel disturbances but had no findings on routine investigations. Three had diarrhoea and passed more than $300 \mathrm{~g}$ loose stool per day and the other three had constipation passing less than $50 \mathrm{~g}$ per day. One of the patients with diarrhoea was investigated a second time, having taken loperamide half an hour before the study. The remaining patients were taking no medication.

Each subject gave their fully informed written consent for the study to be carried out. The protocol was approved by the Ethical Subcommittee of the Sheffield Area Health Authority (Teaching).

After an overnight fast, each subject ate a solid meal $^{2}$ consisting of $120 \mathrm{~g}$ baked beans, $150 \mathrm{~g}$ mashed potato, three Frankfurter sausages and a dessert containing homogenised pineapple in custard (75 g). The total wet weight of the meal was $405 \mathrm{~g}$ and the total calorie intake was estimated to be $536 \mathrm{Kcal}$. Each subject was given $100 \mathrm{ml}$ of water to drink with the meal.

The mashed potato contained $250 \mu \mathrm{Ci}\left(9 \cdot 3 \mathrm{MB}_{\mathrm{q}}\right)$ of radioactive ${ }^{99 \mathrm{~m}}$ Technetium which was complexed with sulphur colloid so that it was not absorbed by the gastrointestinal tract. This was mixed in the water that reconstituted the mashed potato. This isotope has a radioactive half life of six hours and would be expected to be half cleared from the gastrointestinal tract in approximately 50 hours in a normal individual. The radiation exposure was calculated to be between 30 and 40 mrads to the gut and between 10 and 20 mrads to the gonads. This was less than the radioactivity received during an abdominal radiograph (100-200 mrads to the gut; 13.7 mrads to the testes; 146 mrads to the ovaries).

After the meal had been eaten the subject lay supine on an air mattress and the gamma camera (model 400T, Elscint, Israel) was positioned over the abdomen at a distance of about $10 \mathrm{~cm}$ so that the whole of the abdominal cavity could be monitored. Two markers (lead lined plastic bottle tops, $1.5 \mathrm{~cm}$ in diameter and containing cotton wool soaked with $5 \mu \mathrm{Ci}\left(180 \mathrm{~KB}_{\mathrm{q}}\right)$ radioactive ${ }^{99 \mathrm{~m}} \mathrm{Technetium}$ sulphur colloid) were fixed to the skin, one situated in the right hypochondrium at the end of the ninth rib, the other over the left anterior superior iliac spine. These remained in position for the remainder of the study and established anatomical reference points which were used to correct for the movement of the patients when the study was analysed.

The gamma camera was connected to a dedicated minicomputer programmed to record cumulative integrated images of the distribution of radioactivity over consecutive five minute periods for the whole of the study.

Samples of end expiratory air were obtained at the beginning of the study and at 10 minute intervals thereafter using a modified Haldane Priestley tube. ${ }^{6}$ The concentration of hydrogen in each sample was measured by means of an electrochemical detector containing a gas sensitive electrode. ${ }^{7}$ A rise in breath hydrogen concentration is produced by fermentation of unabsorbed sugars (such as the stachyose and raffinose in the baked beans) by colonic bacteria, ${ }^{1}$ and was used to signal the arrival of the meal in the colon.

Each study lasted at least eight hours. Subjects lay supine for all of that period and were only allowed to leave the couch when they needed to urinate.

ANALYSIS OF GAMMA CAMERA RECORDS

This proceeded in several stages. First, the images containing the integrated counts over successive five minute periods were aligned with reference to the two markers in the right hypochondrium and left iliac fossa. This analysis assumed that the subject remained flat on his back for the whole of the study and any movement was in the horizontal plane. Next, the total abdominal region was outlined, excluding the two markers, and the total number of counts in the abdominal cavity was normalised to a constant value. This corrected both for isotope decay and variations due to the relative distance of the source from the detector. Figure 1 indicates the totalised distribution of radioactivity in the abdomen throughout the whole of the study. The position of the stomach was identified at the beginning of the study and an outline drawn around it using a cursor controlled by a joystick. The position of the colon was also identified in the same way in the last few frames of the study and was similarly outlined (Fig. 1). The small intestinal area was generated from the counts outside the regions of the stomach and colon, and curves were drawn of the proportion of total counts in each of these three regions at five minute intervals throughout the study (Figs 2 and 3).

In some studies, overlap existed between the small intestinal and colonic zones and between the gastric and colonic zones. This problem was tackled in the following sequence. ${ }^{8}$ An exponential decay curve was fitted over the curve for gastric emptying to exclude counts in the stomach region produced by counts reaching the transverse colon at the end of 


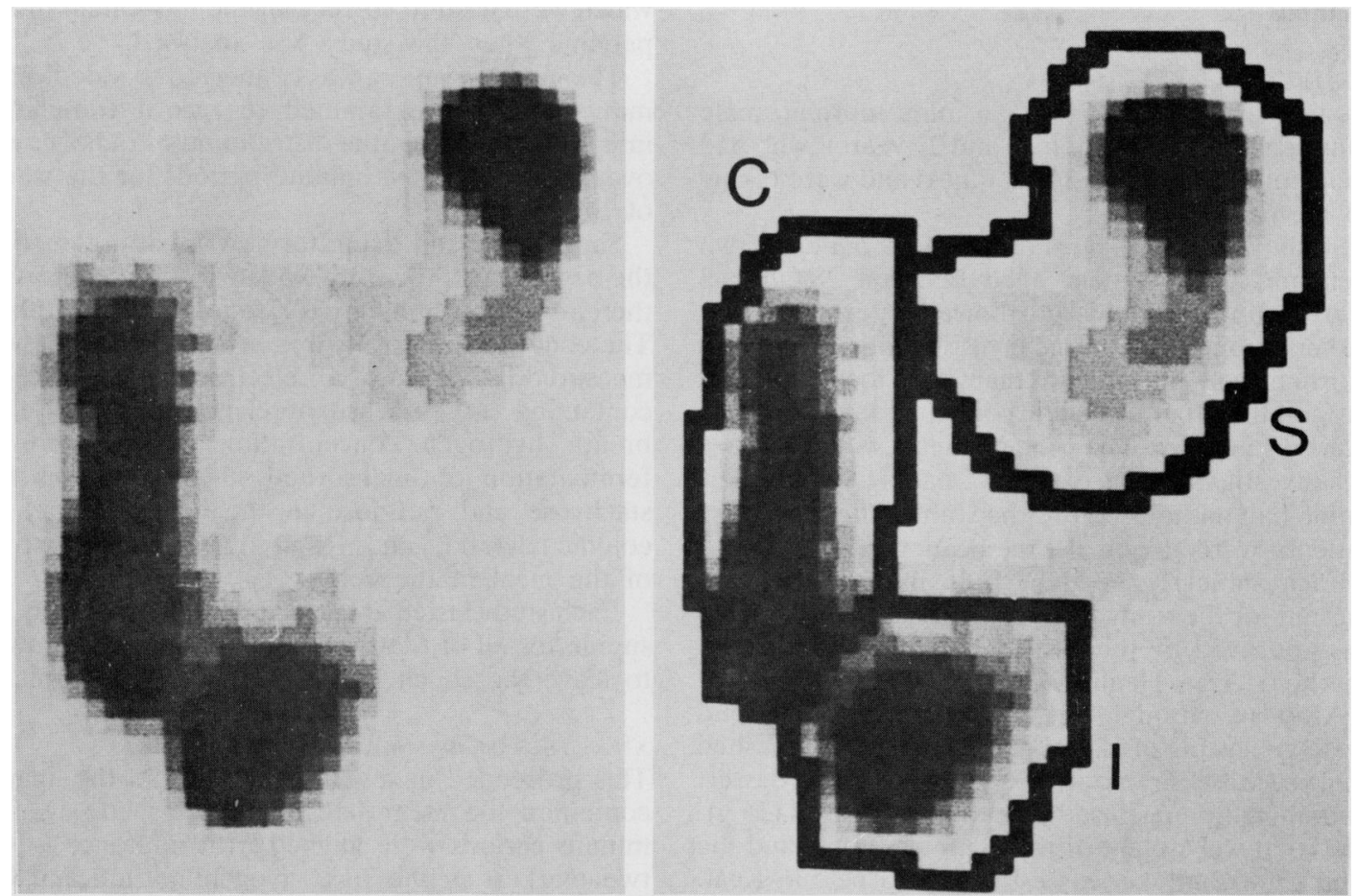

Fig. 1 Integrated distribution of radioactivity in the abdominal cavity throughout the whole study in one typical normal subject. In the images on the left, areas of interest are drawn around the stomach $(S)$ and colon $(C)$ and a region in the pelvis which probably corresponds to the ileum (I).

the study. By subtracting the new gastric curve from the total counts, a curve describing the amount of isotope in the combined small and large intestine was generated. As the first part of the small intestinal curve was the same shape as the first part of the combined small and large intestinal curve and the breath hydrogen did not rise until later in the study, it was assumed that the two curves described entry into the small intestine and any difference between them was merely a scaling factor caused by the loss of small intestinal counts caused by some overlap with the colonic zone. To correct for this, the first part of the small intestinal curve was scaled up until it matched the first part of the combined curve. In practice the scaling factor was always less than $10 \%$. The point at which the two curves began to deviate indicated the point of entry of food residues into the colon. Finally, the new colon curve was generated by subtracting the scaled up small intestinal curve from the combined small and large intestinal curve. The new curves for gastric emptying, small bowel residence and colonic filling were then plotted on a graph and values for the rate of gastric emptying small bowel residence and colonic filling were obtained.

The half time for gastric emptying was determined from the profile of gastric emptying. Values for the onset, $50 \%, 80 \%$, and $100 \%$ colonic filling were obtained from the colonic profiles. Finally, small intestinal residence or contact time was calculated by integrating the area under the intestinal curve and expressed as food hours, where one food hour is equivalent to the residence of all the ingested isotope in the small intestine for one hour. It should be emphasised that the term food hour provides a semiquantitative index of the residence of the radioactive marker in the small intestine. It cannot be used to indicate the amount of food in the small intestine since this depends on the degree of the absorption of the meal.

ESTIMATION OF MEAN TRANSIT TIME

When food enters the small intestine, each particle does not travel down the intestine at the same rate, some particles will travel faster than others. Thus there is no single value for transit time, instead there 


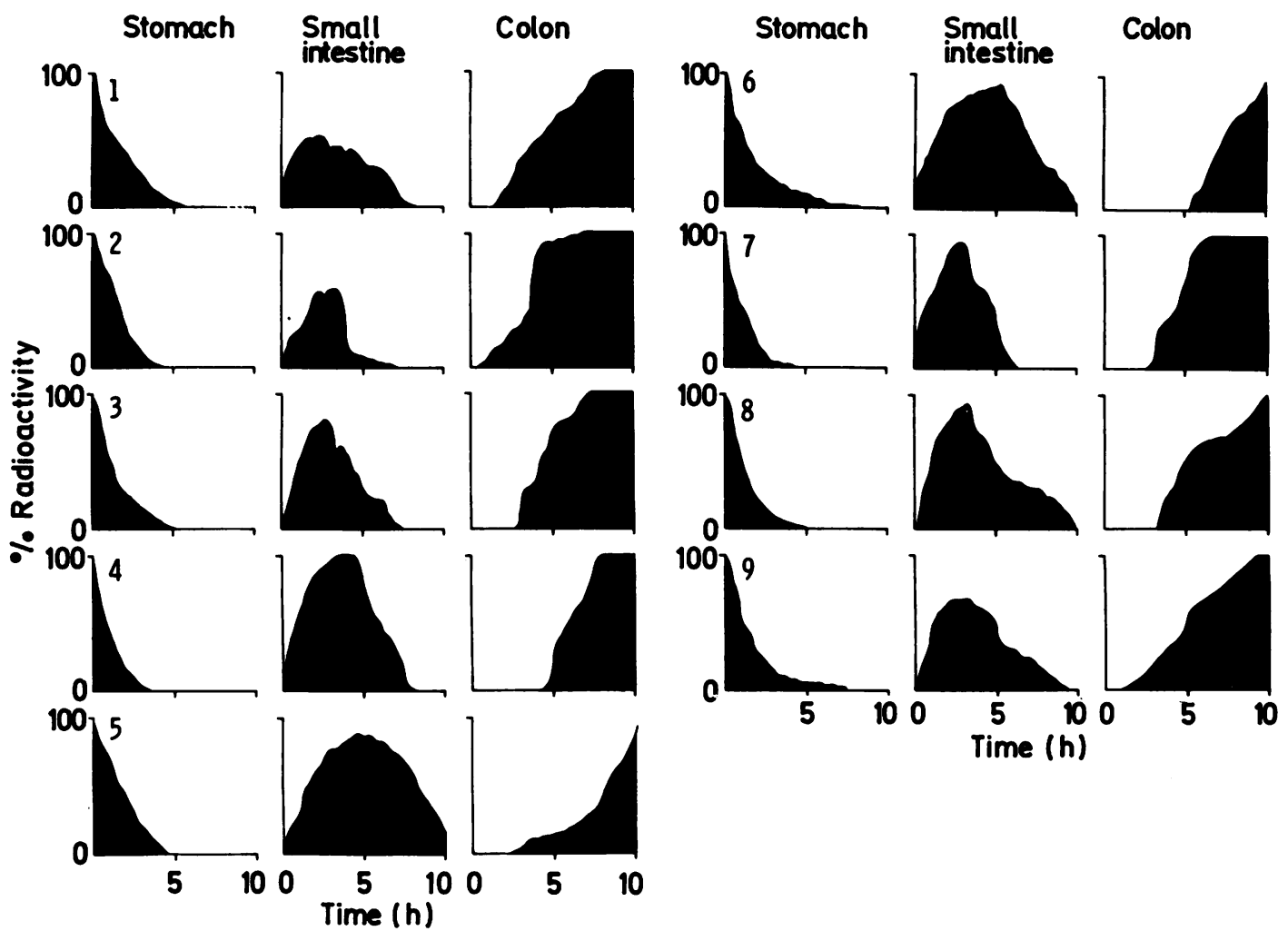

Fig. 2 Profiles for gastric emptying, small bowel residence and colonic filling in nine normal volunteers. The curves were generated by monitoring the progress of a solid meal through the gut using a gamma camera linked to a dedicated minicomputer.

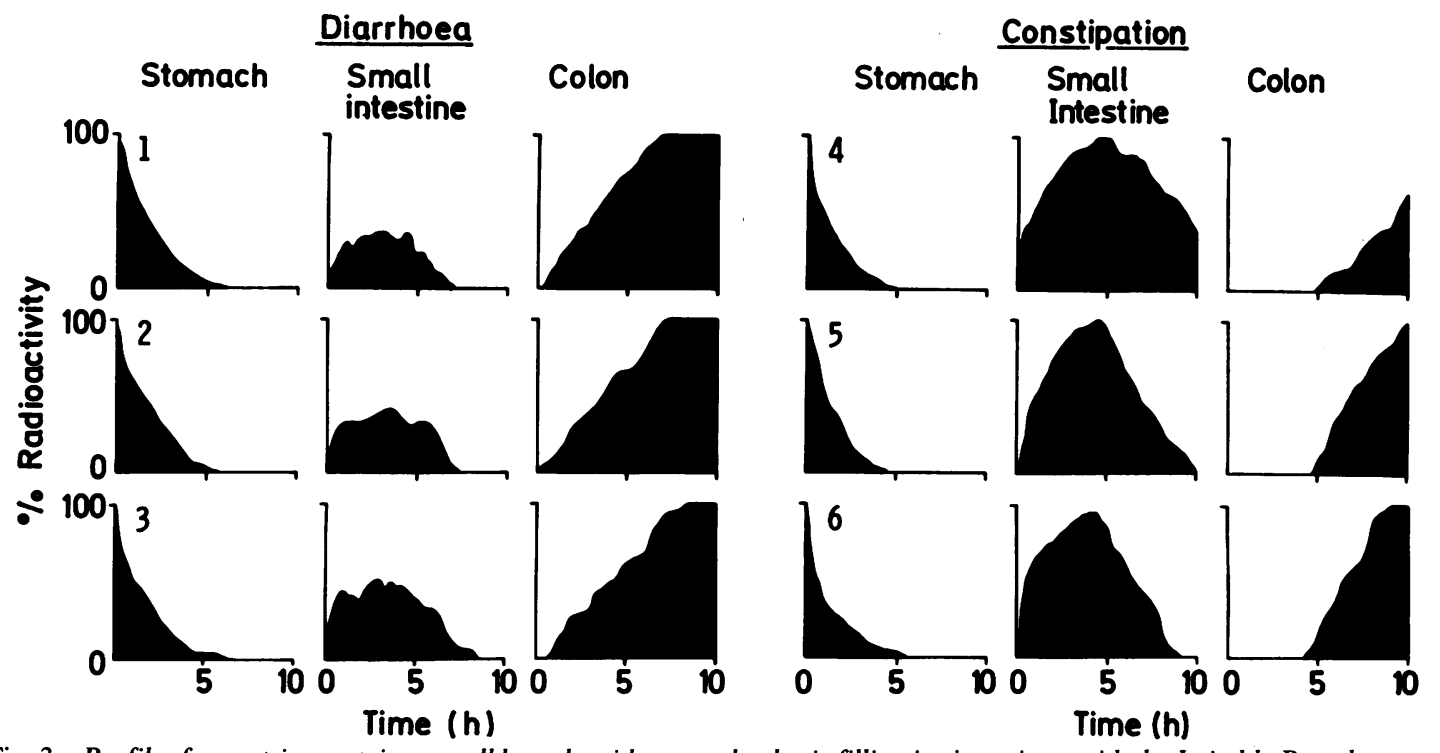

Fig. 3 Profiles for gastric emptying, small bowel residence and colonic filling in six patients with the Irritable Bowel Syndrome. The curves were generated by monitoring the progress of a solid meal through the gut using a gamma camera linked to a dedicated minicomputer. 
is a spectrum of transit times. The rate at which food leaves the intestine can be determined by convolving the input function, the rates at which food enters the small intestine, with the spectrum of transit times. We have determined the input and output rates experimentally. It therefore follows that the transit time spectrum can be determined by deconvolving the rate of colonic filling with the rate of gastric emptying. From this spectrum the value of the mean transit time can be obtained.

$$
\text { MTT }=\frac{t . s(t) d t}{s(t) d t}
$$

where $s(t)$ is the transit time spectrum.

\section{STUDIES IN ILEOSTOMY PATIENTS}

In order to compare the delivery of the residues of the meal from the terminal ileum with the values for colonic filling obtained in our study and to validate the use of the radioactive marker as an index of the emptying of the components of the meal, measurements of small bowel transit time and the composition of ileal effluent were carried out in 14 patients equipped with a terminal ileostomy. ${ }^{9}$

After an overnight fast, each subject ate a solid homogenised meal composed of $100 \mathrm{~g}$ lean ham, $218 \mathrm{~g}$ mashed potato, $100 \mathrm{~g}$ washed baked beans and $25 \mathrm{ml}$ corn oil and labelled with $50 \mu \mathrm{Ci}\left(1.8 \mathrm{MB}_{\mathrm{q}}\right)$ of ${ }^{99 \mathrm{~m}}$ Technetium sulphur colloid (half-life $=6 \mathrm{~h}$ ). ${ }^{9}$

The time taken for the meal to reach the terminal ileum was estimated by determining the cumulative delivery of radioisotope in sequential half hour samples of ileal effluent collected for a period of 24 hours after ingestion of the test meal. In three subjects, the delivery of the marker was compared with the delivery of protein, fat and carbohydrate components of the meal, determined by chemical analysis of each half hour sample.

\section{Results}

\section{STUDIES IN NORMAL SUBJECTS}

\section{Gastric emptying}

The average value for the half time for gastric emptying was $1: 2 \pm 0.3$ hours (mean $\pm S D ; n=9$ ), and there was up to $12 \%$ food (mean $=6 \%$ ) in the stomach by four hours after eating the meal.

\section{Colonic filling}

The colon appeared to fill in linear fashion in most subjects. The average time that food first appeared in the caecum was $2 \cdot 8 \pm 1.5$ hours (SD). Half of the food residues had entered the colon by $5.4 \pm 1.6$ hours, $80 \%$ of the food residues had entered by
$7 \cdot 2 \pm 1.9$ hours, and extrapolating the individual slopes suggested that colonic filling was complete by $8.6 \pm 1.5$ hours. The average profile suggested that approximately one-sixth of the small intestinal residues $(16 \%)$ emptied into the caecum every hour and was very similar to the rate of emptying of the homogenised radiolabelled solid meal from the ileum of patients with terminal ileostomies (Fig. 4), except that the latter commenced later.

\section{Small bowel residence}

Profiles of small bowel residence are shown in Figures 2 and 3 . The initial steep rise in the profile is dominated by gastric emptying. This rises to a peak and then slowly falls as the rate of exit of residues into the caecum exceeds the rate of entry of food from the stomach. The rate of decline increases as the curve becomes more and more dominated by caecal filling with little or no entry of food from the stomach and finally assumes the configuration of the mirror image of the caecal filling curve. The average value for intestinal residence was $3.5 \pm 1 \cdot 4$ food hours.

Totalling the images showed that in six of nine studies the isotope collected at a point in the lower pelvis (Fig. 1) soon after ingesting the meal, and then passed gradually to the colon. This region presumably corresponded to the ileum and suggests that the latter is a region of relative stasis.

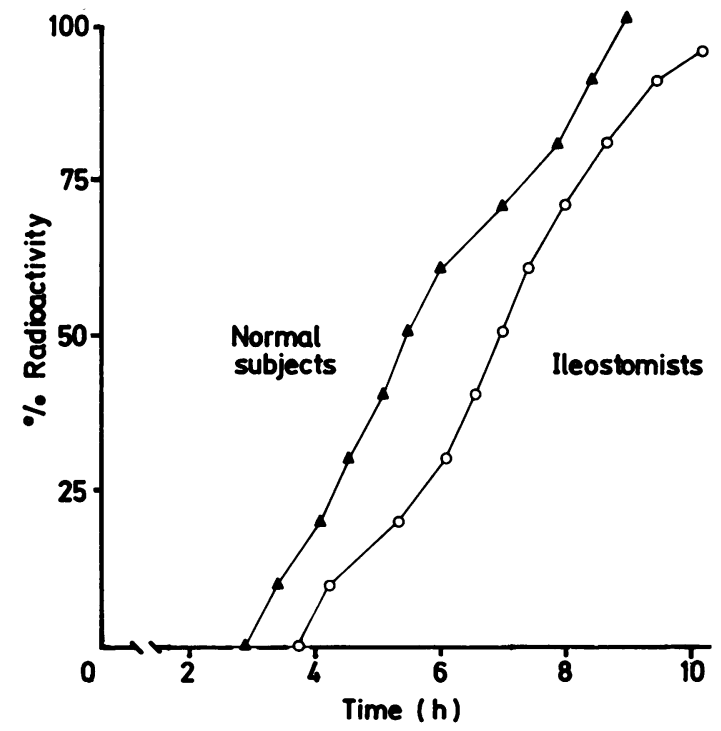

Fig. 4 Average profile of colonic filling in nine normal subjects after ingestion of a solid meal compared with the average profile of ileal emptying found in 14 patients with terminal ileostomies after ingesting a homogenised meal of similar composition. 
Mean transit time

The mean transit time obtained by deconvolving the rates of colonic filling with the rates of gastric emptying was $4 \cdot 0 \pm 1.4$ hours, and was very similar to the value obtained by subtracting the $t_{2}^{1}$ for gastric emptying from the $t_{2}^{1}$ for colonic filling $(4 \cdot 1 \pm 1 \cdot 5$ hours).

\section{Breath hydrogen excretion}

In six of nine normal subjects, breath hydrogen profile consisted of an initial peak of hydrogen that commenced as the meal was ingested and lasted between 20 and 50 minutes before the hydrogen concentration subsided to basal levels, and a secondary rise that lasted until the study was complete. ${ }^{2}$ The initial transient peak of hydrogen concentration occurred before the arrival of radioactivity in the caecum in every case.

STUDIES IN PATIENTS WITH THE IRRITABLE BOWEL SYNDROME

The results from the patients with irritable bowel syndrome are shown in Figures 3 and 5. Patients with diarrhoea tended towards one end of the normal range with early colonic filling, and short small intestinal transit times and residences, whereas patients with constipation tended towards the other end with delayed colonic filling and prolonged small intestine transit times and residences $(p<0.05)$ (Fig. 5).

Administration of loperamide to one patient with diarrhoea delayed the half time for gastric emptying 84 to 108 minutes, delayed the arrival of the meal at the caecum from 10 minutes to 122 minutes, delayed the arrival of $50 \%$ of the meal from 200 to 360 minutes and the arrival of all the meal at the caecum from 424 to 660 minutes. The mean transit time was almost trebled (from 115 to 310 minutes), and the meal residence increased from 1.4 to 1.9 food hours.

\section{CORRELATIONS BETWEEN TRANSIT DATA}

There were significant linear correlations between the mean transit time and the difference between the two half times $(r=0.92)$, the small intestinal residence $(r=0.83)$, the value for the time at which the marker arrived in the colon $(r=0.86)$ and the $\mathrm{t} 50 \%(\mathrm{r}=0.84)$ and $\mathrm{t} 80 \%(\mathrm{r}=0.86)$ for colonic filling but not the $t_{2}^{1}$ for gastric emptying $(r=0.315)$.

There was a highly significant correlation between the half time for colonic filling and intestinal residence $(r=0 \cdot 89)$. There were no significant correlations between the half times for colonic filling

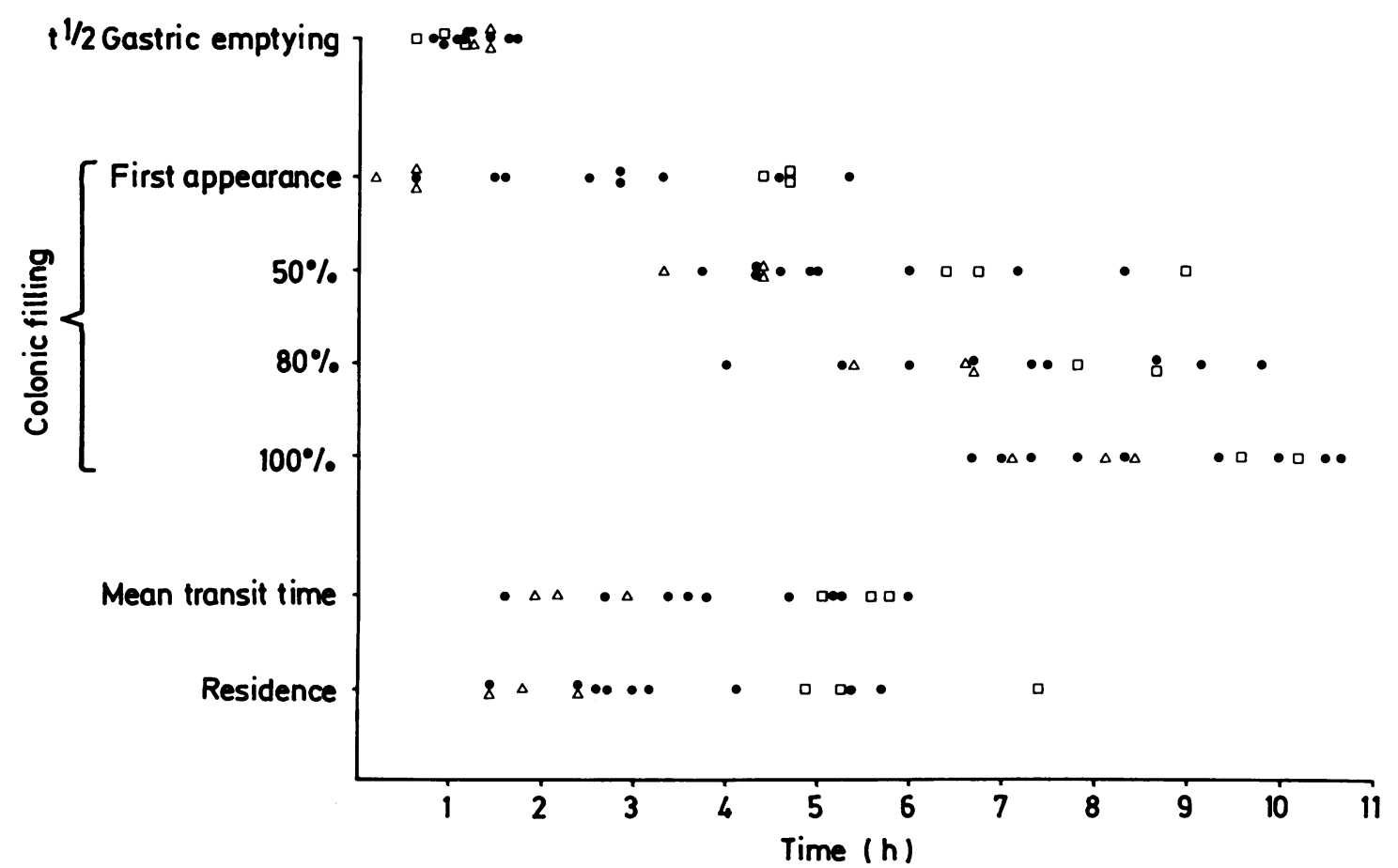

Fig. 5 Values for gastric emptying, colonic filling and mean small bowel transit time and small intestinal residence in normal subjects $(O)$ and patients with irritable bowel syndrome with diarrhoea $(\triangle)$ and constipation $(\square)$. 
and gastric emptying $(\mathrm{r}=0 \cdot 1)$ or between intestinal residence and the half time for gastric emptying $(r=0 \cdot 32)$.

There was a highly significant correlation $(r=0.88 ; p<0.001)$ between the secondary rise in breath hydrogen excretion and the onset of colonic filling.

VALIDATION OF THE MARKER TO DETERMINE TRANSIT OF THE MEAL

In each of the three ileostomists, there were excellent linear correlations between the delivery of the radioactive marker in ileostomy effluent and the delivery of protein $(r=0.99)$, fat $(r=0.98)$ and absorbable carbohydrate $(r=0.99)$.

\section{Discussion}

The technique of labelling the food with a small amount of radioisotope and plotting its progress through the intestine by means of a gamma camera placed over the abdomen, allows quantitative data to be obtained for gastric emptying, for the rate of entry of the residues of the meal into the colon, for the residence of the meal in the small intestine, and for the average transit time of the meal through the small intestine. This potentially useful technique provides the opportunity to study the influence of small bowel residence or exposure time on the absorption of food components, to investigate how gastric emptying or small bowel motor activity influence small bowel transit time and colonic filling and to compare the rate of colonic filling or small bowel transit time in patients with bowel complaints. Although few patients with irritable bowel syndrome could tolerate the study, the results supported our previous conclusions from the breath hydrogen technique that small bowel transit times tended to be short in patients with diarrhoea but long in patients with constipation. ${ }^{10}$

When food enters the small intestine, individual particles travel at widely different rates to the caecum. Thus, strictly speaking, there is no true transit time, but a spectrum of transit times. The values quoted in this study are the mean of the transit time spectrum and are theoretically the most representative values for transit time. In most subjects, however, they are very similar to values obtained for the difference in the half times for gastric emptying and colonic filling although they are longer than the values for the onset of colonic filling. We found that the small bowel transit time and the residence of a meal in the small intestine were correlated with the rate of colonic filling but not with the rate of gastric emptying. This result confirms the results of studies carried out using a single scintillation detector to measure gastric emptying. ${ }^{11}$

The radiation exposure during this study is relatively low, the marker substances do not interfere with the normal passage of food through the gut, and the definition is good enough to recognise the stomach, small intestine, ileum, and colon and plot the passage of the food through or into these areas. Although the radioactive marker is mixed with the liquid which reconstitutes the mashed potato, we have been unable to show any difference in the rate of emptying of the meal used in this study from the stomach whether the marker was in the water that reconstituted the mashed potato or whether it was incorporated in chicken liver. ${ }^{2}$ This would support the results of Hinder and Kelly, ${ }^{12}$ who showed that the solid and liquid components empty at the same rate from meals of homogeneous consistency (such as our meal) but at different rates from meals that are composed of well separated solid and liquid phases (such as the steak and water meal used by Meyer and his colleagues. ${ }^{13}$ Moreover, when a radioactive marker was incorporated in the fluid that made up the mashed potato, and fed in a solid meal to patients with ileostomies, the delivery of the marker from the distal ileum was closely correlated with the delivery of the carbohydrate, fat and protein components of the meal.

The observation that profiles of colonic filling determined in normal subjects by the gamma camera were very similar to the profiles of ileal emptying in patients with ileostomies suggests that both techniques were measuring the same phenomenon, despite the slightly different meals and the fact that the ileostomists were ambulant. The slower passage of the meal through the terminal ileum in ileostomists may suggest that the presence of ileocaecal valve does not delay ileal emptying.

The derivation of a profile for colonic filling relies on the assumption that nearly all of the meal will be in the proximal colon by eight hours after ingestion and the colonic profile will be easily recognised. As the images are two-dimensional, however, and the definition relatively poor compared with radiology, a loop of small bowel overlapping the colonic zone could appear as an early rise in colonic filling. The method we used to correct for overlap between the small intestine and colon assumed (a) that the original identification of the colonic zone was accurate and (b) that the distribution of the isotope in the small intestinal zone was reasonably uniform so that a relatively fixed proportion of isotope in the small intestine would overlap the colon throughout the study. This assumption is likely to be correct for a loop of proximal small bowel overlapping the colon, but may not always be true for an overlapping 
loop of terminal ileum. Despite this potential problem, we found there was a significant correlation between the entry of the radioisotope into the colon and the secondary increase in breath hydrogen concentration; in six of nine normal subjects and four of six patients with the irritable bowel syndrome the rise in breath hydrogen excretion occurred within 20 minutes of the arrival of radioactivity in the caecum. In the remaining subjects, the increase in colonic radioactivity preceded the rise in breath hydrogen by up to 90 minutes. This suggested an overlapping loop of terminal ileum since we have previously shown that a rise in breath hydrogen can occur within two minutes of direct caecal injection of as little as $0.5 \mathrm{~g}$ carbohydrate.$^{14}$ In future studies, it may be possible to correct for overlapping loops of ileum by noting the onset of the breath hydrogen response. Modern gamma cameras have the facility of taking three dimensional images and will greatly assist the accurate discrimination of gastrointestinal regions.

Overlap between the stomach and small intestine was no problem because when the subject was lying flat, the gastric region always appeared well separated from the small intestinal region (possibly by the presence of the transverse colon or because of dilution of the marker and rapid progress in the upper small intestine). Similarly, overlap between the stomach and colon could be easily detected because this occurred at the end of the study when nearly all the meal residues had left the stomach.

In order to collect data from normal subjects it was necessary for our volunteers to lie supine for up to eight hours. This may have induced an element of stress in some individuals. As some subjects found it difficult to keep perfectly still for the whole of the study (up to eight hours), individual five minute frames had to be individually aligned using fixed sources attached to the abdominal wall. While this corrected for movements in a horizontal plane, it did not allow for twisting movements so that the supine body is either resting more on one side than the other. Although movement did not present insurmountable problems in analysis, future studies could use an adjustable frame or body shell to fix the subjects in the same position for each period of counting. Using such a device, it would be possible to count for short periods of time, for example two minutes every 20 minutes, and allow the subject to get off the couch in between counting periods. This would make the procedure simple and tolerable for patients and speed up the analysis of the data.

The data were analysed on the assumption that the isotope was always the same distance from the detector. This was not altogether true because the small intestine is closer to an anteriorly placed detector than the stomach and caecum and therefore the isotope moved towards and then away from the detector as it passed down the gastrointestinal tract. This phenomenon resulted in an apparent increase in the amount of isotope present in the abdomen in the middle of the study, when most of the isotope was in the small intestine, than at the beginning or at the end. We attempted to correct for this phenomenon and for isotope decay by normalising the radioactive counts in each frame to the same total. This process, however, introduced an error in as much as each region was treated in the same way. For example, if the total abdominal counts become lower because food moves from small intestine to colon, the colon counts should be scaled up much more than the small intestinal counts. This is a formidable task, however, because it is difficult to choose appropriate differential scaling factors for each region in each individual. The present practice of normalisation means that the small bowel residence tends to be overestimated and the degree to which this occurs may vary according to the build of the subject. This problem may be circumvented by using two gamma cameras, one placed anterior and the other posterior to the abdomen,${ }^{15}$ or by using one camera and collecting alternate anterior and posterior images by moving the camera around the body. Using this method, the area of the intestine that is closer to one camera head will be further from the other and errors will cancel out.

Although the radiation exposure is relatively low, we felt this factor precluded multiple studies in our normal volunteers, and prevented us from assessing the reproducibility of the measurements. A reduction in dose would have led to poor definition and difficulty in differentiating between different regions of the gut. It is possible that multiple studies would be considered acceptable in other centres.

If we can accept or deal with the limitations discussed above, then this technique offers a potentially useful means of measuring small bowel transit time studying small intestinal residence and the dynamics of caecal filling in man and the effects that disease has on these indices.

\section{References}

1 Bond $\mathrm{JH}$, Levitt MD. Investigation of small bowel transit time in man utilising pulmonary hydrogen $\left(\mathrm{H}_{2}\right)$ measurements. J Lab Clin Med 1974; 85: 546-59.

2 Read NW, Miles CA, Fisher D, et al. Transit of a meal through the stomach, small intestine and colon in normal subjects and its role in the pathogenesis of diarrhoea. Gastroenterology 1980; 79: 1276-82.

$3 \mathrm{Kim}$ SJ. Transit time in the normal small bowel study. Am J Roentgenol 1968; 104: 522-9. 
4 Lonnerblad L. Transit time through the small intestine. A roentgenologic study on normal variability. Acta Radiol suppl 1951; 88: 1 .

5 Mattson O, Perman G, Lagerlof $H$. The small intestine transit time with a physiologic contrast medium. Acta Radiol (Stockh) 1960; 54: 334-44.

6 Metz G, Gassull MA, Leeds AR, Blendis LM, Jenkins DJA. A simple method of measuring breath hydrogen in carbohydrate malabsorption by end-expiratory sampling. Clin Sci Mol Med 1976; 50: 237-40.

7 Bergman I, Coleman JE, Evans D. A simple gas chromatograph with an electrochemical detector for the measurement of hydrogen and carbon monoxide in the parts per million range, applied to exhaled air. Chromatographia 1975; 8: 581-3.

8 Read NW, Al-Janabi MN, Bates TE, Barber DC. Effect of gastrointestinal intubation on the passage of a solid meal through the stomach and small intestine in humans. Gastroenterology 1983; 84: 1568-72.

9 Holgate AM, Read NW. Relationship between small bowel transit time and absorption of a solid meal. Influence of metoclopramide, magnesium sulfate and lactulose. Dig Dis Sci 1983; 28: 812-9.
10 Cann PA, Read NW, Brown C, Hobson N, Holdsworth $\mathrm{CD}$. Irritable bowel syndrome: relationship of disorders in the transit of a single solid meal to symptom patterns. Gut 1983; 24: 405-11.

11 Read NW, Cammack J, Edwards C, Holgate AM, Cann PA, Brown C. Is the transit time of a meal through the small intestine related to the rate at which it leaves the stomach? Gut 1982; 23: 824-8.

12 Hinder RA, Kelly KA. Canine gastric emptying of solids and liquids. Am J Physiol 1977; 233: E335-40.

13 Meyer JH, MacGregor IL, Gueller R, Martin P, Cavalieri R. ${ }^{99 \mathrm{~m}} \mathrm{Tc}$-tagged chicken liver as a marker of solid food in the human stomach. Am J Dig Dis 1976; 21: 296-304.

14 Read NW, Al-Janabi MN, Bates TE, et al. In interpretation of the breath hydrogen profile obtained after ingesting a solid meal containing unabsorbable carbohydrate. Gut (In press).

15 Ackermans LMA, Hoekstra A, Renooij W, Haarman HJThM. Measuring gastric emptying with two gamma cameras. In: Ackermans LMA, Johnson AT, Read NW eds. Gastric and gastroduodenal motility. Eastbourne: Praeger 1984: 158-62. 